From the Editors

This issue of RENSIT is devoted to the works of the Fryazino Branch

of the Kotelnikov Institute Radioengineering and Electronics of RAS \%

\title{
RESEARCH IN THE FRYAZINO BRANCH OF THE KOTELNIKOV INSTITUTE OF RAS ON RADIOLOCATION AND INFORMATION TECHNOLOGIES
}

\section{Lev E. Nazarov, Vladimir M. Smirnov}

Kotelnikov Institute of Radioengineering and Electronics of RAS, Fryazino branch, http:// fire.relarn.ru Fryazino 141190, Moscow region, Russian Federation

levnaz2018@mail.ru,vsmirnov@sunclass.ire.rssi.ru

Abstract. The information on the publication of a number of works in the Fryazino branch of the Kotelnikov Institute (FIRE) of RAS on Radiolocation and Information Technologies, dedicated to the 160th anniversary of the birth of the outstanding Russian scientist, inventor of the radio Alexander S. Popov. A selection of publications presents inevitably to the limited range of work carried out in FIRE on this topics, however, it reflects their diversity and level.

Keywords: radar, radio sounding of planets, radiophysical technologies, information technologies

PACS 01.10 VF

Received 23.04.2019, accepted 26.04.2019

RENSIT, 2019, 11(1):3-4

DOI: $10.17725 /$ rensit.2019.11.003

In 2019, the 160th anniversary of the birth of the outstanding Russian scientist, radio inventor Alexander Stepanovich Popov (1859-1905) is celebrated. Creation and public demonstration of A.S. On May 7, 1895, the Popov wireless radio system laid the foundation for the emergence and development of many completely new ideas and scientific directions on the theory and methods of transmitting and processing information, on radiolocation, on studying radio channels of transmission, on radiophysical methods of remote sensing of the earth's surface, on radiophysical technologies of research planets and the sun. Each of these areas includes a complex independent set of scientific and technical problems, the solutions of which are relevant to the present time when creating terrestrial and satellite communication systems based on developed mathematical theories; in the development and creation of radar systems, in particular, remote sensing systems (Earth remote sensing) and systems of radiophysical studies of the planets of the solar system; with the development of information technology. The structural appearance of these systems implements the approach of integrating and combining a number of functional components of subsystems of various purposes and levels - the transfer of information in digital format using the methods of noise protection; archiving, storage and processing of information; adaptability in order to reduce the distorting effects of transmission channels of various physical nature; probing technologies and techniques for the thematic processing of remote sensing data and radiophysical studies of the planets; software and hardware implementation of systems, etc.

The activities of the divisions of radio engineering FIRA them. VA Kotelnikov of the Russian Academy of Sciences from the moment of its formation to the present is closely connected with the solution of these complex problems. Scientific radio engineering studies currently being conducted are based on the fundamental work of the Institute's staff, who conceptually formed, headed and coordinated relevant thematic areas (V. Kotelnikov, N. Armand, G. M. Petrov, A. E. Basharinov, A. .M. Shutko, V.M. Polyakov, A.V. Sokolov, O.I. Yakovlev, A.G. Paveliev, N.A. Savich, V.A. Andrianov, Yu.G. Tishchenko and many others).

This issue of the journal presents the work of the FIRE employees, whose scientific activities are related to the designated radio engineering topics. 
Radiolocation and radio sounding of planets is an important and basic direction developed in FIRE. In the release of this direction is represented by two works.

In the article Gavrik A.L. with co-authors a number of tasks were formulated with co-authors on the subject of radio occultation experiments in the planned Venus-D mission, in particular, studying the propagation of wave processes from the atmosphere to the ionosphere of the planet Venus.

In the article Yushkova O.V. with co-authors, the problems of the bistatic radiolocation of the moon from the spacecraft were formulated with the aim of studying the physical parameters of its surface and the subsurface structure of the soil.

Earth remote sensing is a traditional and at the same time key research area of FIRE. One of its branches - active and passive sensing of earth covers - is presented in the issue with articles that show the results of studies of the most significant environments for human existence - vegetation and snow cover, the aquatic environment, and monitoring of emergency areas.

In the work of Rodionova N.V. outlines the results of using radar data from the Sentinel-1 satellite to determine the seasonal state of soil covers. The possibility of identification of thawed and frozen soils of Alaska in the upper $5 \mathrm{~cm}$ layer is shown, which is confirmed by the data of ground stations.

The features of seasonal variations in the radiophysical properties of the vegetation covers of the Transbaikalia according to the PALSAR cosmic polarimetric radar, including the reflective properties of trees and the dominant mechanisms of scattering of the probing signal by elements of crowns and trunks, are studied in the article Zakharova L.N. and Zakharov A.I. The significant effect of seasonal variations in air temperature on the signal reflection level and the nature of the dominant scattering mechanism is shown, which should be taken into account when building automated algorithms for classifying boreal forest types and evaluating their biophysical parameters.

The possibilities of phase methods, which form the basis of radar interferometry, for monitoring areas of emergency situations are disclosed in the article by A.I. Zakharov and Zakharova L.N. on the example of the processing of radar images of the landslide coastal slope of the Bureya River from the Sentinel-1 satellite.

In the work of Golunov V.A. The results of theoretical and experimental radiophysical studies of the features of volume scattering and the formation of thermal radiation in dry, layered snow are presented.

In the work of Evtikhov M.G. and Arzamastseva G.V. algorithms have been developed for calculating the dependences of the reflection and transmission coefficients of free plates, which are applied in the L-range for quasi-anisotropic ice.

The scientific direction related to information technologies and developed at FIRE determines the creation and development of the theory and methods of transmitting information through various physical channels through the development of methods for processing, storing and archiving information, including images of remote sensing data in digital format, creating mathematical models and specialized software that implements the developed computational algorithms. In the issue this direction is represented by the work of Kokoshkin A.V. with co-authors (results are given on the development and computer simulation of an interpolation algorithm for solving the problem of achieving superresolution of images in a digital format) and the works of Nazarov L.E. with co-authors (the results of theoretical and experimental studies of satellite radio lines regarding the estimation of energy losses due to the influence of signal fading (scintillation) and due to the influence of the Earth's ionosphere as a dispersion medium with respect to the propagation of signals in free space are given. 